\begin{tabular}{|c|c|}
\hline Title & Combinatorics of certain abelian Lie group arrangements and chromatic quasi-polynomials \\
\hline Author(s) & T an Nhat Tran; Y oshinaga, Masahiko \\
\hline Citation & $\begin{array}{l}\text { Journal of combinatorial theory. Series A, 165, 258.272 } \\
\text { https://doi.org/10.1016/.j.jcta.2019.02.003 }\end{array}$ \\
\hline Issue Date & $2019-07$ \\
\hline Doc URL & http:/hdl.handle.net/2115/80475 \\
\hline Rights & $\begin{array}{l}\text { O2019. This manuscript version is made available under the CC-BY-NC-ND } 4.0 \text { license } \\
\text { http://creativecommons.org/icenses/by-nc-nd/4.0/ }\end{array}$ \\
\hline Rights(URL) & https://reativecommons.org/icenses/by-nc-nd/4.0/ \\
\hline Type & article (author version) \\
\hline File Information & Constituent11.pdf \\
\hline
\end{tabular}

Instructions for use 


\title{
COMBINATORICS OF CERTAIN ABELIAN LIE GROUP ARRANGEMENTS AND CHROMATIC QUASI-POLYNOMIALS
}

\author{
TAN NHAT TRAN AND MASAHIKO YOSHINAGA
}

\begin{abstract}
The purpose of this paper is twofold. Firstly, we generalize the notion of characteristic polynomials of hyperplane and toric arrangements to those of certain abelian Lie group arrangements. Secondly, we give two interpretations for the chromatic quasi-polynomials and their constituents through subspace and toric viewpoints.
\end{abstract}

\section{INTRODUCTION}

When a finite list $\mathcal{A}$ of integer vectors in $\mathbb{Z}^{\ell}$ is given, we may naturally associate to it a hyperplane arrangement $\mathcal{A}(G)$ in the vector space $G^{\ell}$ with $G=\mathbb{R}$ or $\mathbb{C}$, and a toric arrangement $\mathcal{A}(G)$ in the torus $G^{\ell}$ with $G=\mathbb{S}^{1}$ or $\mathbb{C}^{\times}$. The study of a hyperplane/toric arrangement typically goes along with the study of its characteristic polynomial as the polynomial carries combinatorial and topological information of the arrangement (e.g., [OS80], [OT92], [Loo93], [DCP05]). Many attempts have been made in order to compute and to make a broader understanding the characteristic polynomials (e.g., [Ath96], [BS98], [KTT08], [ERS09], [Law11], [Moc12], [DM13], [BM14], [Yos18b], [Yos18a]). One of the first and classical ways is to define a polynomial in more than one variable which specializes to the characteristic polynomial. It is well-known that the characteristic polynomial of any hyperplane arrangement gets generalized to the Tutte polynomial [Tut54], going back to Whitney (e.g., [Sta07, Theorem 2.4]). More recently, an arithmetical generalization of the ordinary Tutte polynomial, the arithmetic Tutte polynomial was introduced [Moc12] to extend the analysis on the toric case. For another interesting way of generalizing the polynomials, we would encode the information of the characteristic polynomials of these arrangements in a single quasi-polynomial, the characteristic quasipolynomial of $\mathcal{A}$ [KTT08]. This quasi-polynomial was defined to evaluate the cardinality of the complement of the q-reduced arrangement $\mathcal{A}\left(\mathbb{Z}_{q}\right)$ in

Date: February 4, 2019.

Key words and phrases. $G$-Tutte polynomial, chromatic quasi-polynomial, characteristic quasi-polynomial, constituent, abelian Lie group arrangement. 
$\mathbb{Z}_{q}^{\ell}$. Then the characteristic polynomials of the hyperplane and toric arrangements coincide with the first and the last constituents of the characteristic quasi-polynomial, respectively ([KTT08], [LTY17]).

In a more general setting, all of the concepts mentioned above can be redefined through a finite list $\mathcal{A}$ of elements in a finitely generated abelian group $\Gamma$. The notions of hyperplane, toric and $q$-reduced arrangements are unified by the notion of $G$-plexifications $\mathcal{A}(G)$ [LTY17], which are defined by means of group homomorphisms from $\Gamma$ to certain abelian groups $G$. The $G$-plexifications when $G=F \times\left(\mathbb{S}^{1}\right)^{p} \times \mathbb{R}^{q}$, with $F$ is a finite abelian group are (non-trivial) examples of arrangements of abelian Lie groups. In [LTY17], their topologies provided that $q>0$ have been described by the $G$-characteristic polynomials. Studying problems related to this specific class of abelian Lie group arrangements is a source of our interest and suffices the purpose of generalizing the previous concepts. The first result of the paper is obtained through the motivation of giving a combinatorial framework that describes the intersection patterns of $\mathcal{A}(G)$. Insprired by the pioneered work in [Moc12], we will associate to $\mathcal{A}(G)$ intersection posets and prove that its partial and total characteristic polynomials are also expressible in terms of the $G$-characteristic polynomials (Section 3 ).

The cardinality of the complement of $\mathcal{A}\left(\mathbb{Z}_{q}\right)$ appeared as an analogue of the chromatic polynomial defined on a graph, and is called the chromatic quasi-polynomial [BM14]. We are also interested in a question that given a constituent of a chromatic polynomial how we can describe it in connection with arrangement characteristic polynomials? Less is known, except for the first and the last. Some attempts were made to describe certain classes of the constituents appeared in [Tra18], [DFM17, §10.3.3]. The second result of the paper is two complete interpretations for the constituents through subspace and toric viewpoints. The subspace interpretation is obtained from the combinatorics of $\mathcal{A}\left(\mathbb{R}^{\operatorname{dim}(G)} \times \mathbb{Z}_{k}\right)$, while the toric interpretation is obtained from the arithmetics of $\mathcal{A}\left(\mathbb{S}^{1}\right)$ (or $\mathcal{A}\left(\mathbb{C}^{\times}\right)$) by appropriately extracting its intersection poset (Section 4).

The remainder of the paper is organized as follows. In Section 2, we recall definitions and basic facts of the generalized toric arrangements, $G$ plexifications with the associated $G$-Tutte polynomials, and chromatic quasipolynomials. In Section 3, we define for $\mathcal{A}(G)$ with $G=F \times\left(\mathbb{S}^{1}\right)^{p} \times \mathbb{R}^{q}$ the total and partial intersection posets, and express the corresponding characteristic polynomials in terms of $G$-characteristic polynomials (Theorem 3.7, Corollary 3.8). In Section 4, we obtain the subspace interpretation (Corollary 4.1) for every constituent of a chromatic quasi-polynomial immediately from the preceding section. Then we obtain reciprocity laws for the constituents with the aid of the reciprocity laws for integral arrangements (formula (4.2)). The toric interpretation (Theorem 4.7) is derived from an 
important arithmetical result on the intersection poset (Lemma 4.5). As a consequence, we provide another proof for the Chen-Wang's comparison of the coefficients of the chromatic quasi-polynomial.

\section{PRELIMINARIES}

Let us first fix some definitions and notations throughout the paper. Let $\Gamma$ be a finitely generated abelian group, and let $\mathcal{A} \subseteq \Gamma$ be a finite list (multiset) of elements in $\Gamma$. For each sublist $\mathcal{S} \subseteq \Gamma$, we denote by $r_{\mathcal{S}}$ the rank (as an abelian group) of the subgroup $\langle\mathcal{S}\rangle \leq \Gamma$ generated by $\mathcal{S}$. Given a group $K$, denote by $K_{\text {tor }}$ the torsion subgroup of $K$. Denote $\mathcal{S}^{\text {tor }}:=\mathcal{S} \cap \Gamma_{\text {tor }}$.

2.1. Generalized toric arrangements. Let $\left(\mathcal{P}, \leq_{\mathcal{P}}\right)$ be a finite poset. The Möbius function $\mu_{\mathcal{P}}$ of $\mathcal{P}$ is the function $\mu_{\mathcal{P}}: \mathcal{P} \times \mathcal{P} \longrightarrow \mathbb{Z}$ defined by

$$
\mu_{\mathcal{P}}(a, b):=\left\{\begin{array}{l}
0 \quad \text { if } a ⿱_{\mathcal{P}} b, \\
1 \quad \text { if } a=_{\mathcal{P}} b, \\
-\sum_{a \leq c<b} \mu_{\mathcal{P}}(a, c) \quad \text { if } a<_{\mathcal{P}} b .
\end{array}\right.
$$

A poset $\mathcal{P}$ is said to be ranked if for every $a \in \mathcal{P}$, all maximal chains among those with $a$ as greatest element have the same length, denoted this common number by $\operatorname{rk}_{\mathcal{P}}(a)$.

Now we briefly recall what has been known on combinatorics of generalized toric arrangements following $[\operatorname{Moc} 12, \S 5]$. Set $T:=\operatorname{Hom}(\Gamma, G)$ with $G$ is either $\mathbb{S}^{1}$ or $\mathbb{C}^{\times}$. Each $\alpha \in \mathcal{A}$ determines the subvariety of $T$ as follows $H_{\alpha}:=\{\varphi \in T \mid \varphi(\alpha)=1\}$. The collection $\mathcal{T}(\mathcal{A}):=\left\{H_{\alpha} \mid \alpha \in \mathcal{A}\right\}$ is called the generalized toric arrangement defined by $\mathcal{A}$ on $T$. In particular, when $\Gamma$ is free, $T$ is a torus and $\mathcal{T}(\mathcal{A})$ is called the toric arrangement. To describe the combinatorics of $\mathcal{T}(\mathcal{A})$, we associate to it an intersection poset $L_{\mathcal{T}(\mathcal{A})}$, which is the set of all the connected components of all the intersections of the subvarieties $H_{\alpha}$. The poset $L_{\mathcal{T}(\mathcal{A})}$ is ranked by the dimension of its elements (layers). The combinatorics is encoded in the characteristic polynomial defined by

$$
\chi_{\mathcal{A}}^{\text {toric }}(t):=\sum_{\mathcal{C} \in L_{\mathcal{T}(\mathcal{A})}} \mu\left(T^{\mathcal{C}}, \mathcal{C}\right) t^{\operatorname{dim}(\mathcal{C})},
$$

where $T^{\mathcal{C}}$ is the connected component of $T$ that contains $\mathcal{C}$. To compute $\chi_{\mathcal{A}}^{\text {toric }}(t)$ (in the same way as the Whitney's theorem showing how the characteristic polynomial of a hyperplane arrangement is computed by the Tutte polynomial), Moci introduced the arithmetic Tutte polynomial

$$
T_{\mathcal{A}}^{\text {arith }}(x, y):=\sum_{\mathcal{S} \subseteq \mathcal{A}} \#(\Gamma /\langle\mathcal{S}\rangle)_{\text {tor }}(x-1)^{r_{\mathcal{A}}-r_{\mathcal{S}}}(y-1)^{\# \mathcal{S}-r_{\mathcal{S}}} .
$$


Theorem 2.1 ([Moc12]). If $\Gamma$ is free and $0_{\Gamma} \notin \mathcal{A}$ (or even if $\Gamma$ is arbitrary with $\left.\mathcal{A}^{\text {tor }}=\emptyset\right)$, then

$$
\chi_{\mathcal{A}}^{\text {toric }}(t)=(-1)^{r_{\mathcal{A}}} \cdot t^{r_{\Gamma}-r_{\mathcal{A}}} \cdot T_{\mathcal{A}}^{\text {arith }}(1-t, 0) .
$$

2.2. $G$-plexifications. Let $G$ be an arbitrary abelian group. We recall the notions of $G$-plexifications and $G$-Tutte polynomials of $\mathcal{A}$ following [LTY17, $\S 3]$. We regard $T=\operatorname{Hom}(\Gamma, G)$ as our total group. For each $\alpha \in \mathcal{A}$, we define the $G$-hyperplane associated to $\alpha$ as follows:

$$
H_{\alpha, G}:=\{\varphi \in T \mid \varphi(\alpha)=0\} \leq T
$$

Then the G-plexification $\mathcal{A}(G)$ of $\mathcal{A}$ is the collection of the subgroups $H_{\alpha, G}$

$$
\mathcal{A}(G):=\left\{H_{\alpha, G} \mid \alpha \in \mathcal{A}\right\} .
$$

The $G$-complement $\mathcal{M}(\mathcal{A} ; \Gamma, G)$ of $\mathcal{A}(G)$ is defined by

$$
\mathcal{M}(\mathcal{A} ; \Gamma, G):=T \backslash \bigcup_{\alpha \in \mathcal{A}} H_{\alpha, G}
$$

In what follows, we assume further that $G$ is torsion-wise finite i.e., $G[d]:=\{x \in G \mid d \cdot x=0\}$ is finite for all $d \in \mathbb{Z}_{>0}$. The $G$-multiplicity $m(\mathcal{S} ; G)$ for each $\mathcal{S} \subseteq \mathcal{A}$ is defined by

$$
m(\mathcal{S} ; G):=\# \operatorname{Hom}\left((\Gamma /\langle\mathcal{S}\rangle)_{\text {tor }}, G\right)
$$

\section{Definition 2.2.}

(1) The $G$-Tutte polynomial $T_{\mathcal{A}}^{G}(x, y)$ of $\mathcal{A}$ is defined by

$$
T_{\mathcal{A}}^{G}(x, y):=\sum_{\mathcal{S} \subseteq \mathcal{A}} m(\mathcal{S} ; G)(x-1)^{r_{\mathcal{A}}-r_{\mathcal{S}}}(y-1)^{\# \mathcal{S}-r_{\mathcal{S}}}
$$

(2) The $G$-characteristic polynomial $\chi_{\mathcal{A}}^{G}(t)$ of $\mathcal{A}$ is defined by

$$
\chi_{\mathcal{A}}^{G}(t):=(-1)^{r_{\mathcal{A}}} \cdot t^{r_{\Gamma}-r_{\mathcal{A}}} \cdot T_{\mathcal{A}}^{G}(1-t, 0) .
$$

Proposition 2.3. The leading coefficient of $\chi_{\mathcal{A}}^{G}(t)$ equals $\# \mathcal{M}\left(\mathcal{A}^{\text {tor }} ; \Gamma_{\text {tor }}, G\right)$.

Various specializations of the $G$-plexifications and $G$-Tutte polynomials have appeared in the literature which we refer the reader to [LTY17] for more details. In particular, the hyperplane arrangements and generalized toric arrangements are $G$-plexifications by viewing $G=\mathbb{R}($ or $G=\mathbb{C})$ and $G=\mathbb{S}^{1}\left(\right.$ or $\left.G=\mathbb{C}^{\times}\right)$, respectively. 
2.3. Chromatic quasi-polynomials. For each $\mathcal{S} \subseteq \mathcal{A}$, by the Structure Theorem, we may write $\Gamma /\langle\mathcal{S}\rangle \simeq \bigoplus_{i=1}^{n_{\mathcal{S}}} \mathbb{Z}_{\mathcal{S}_{\mathcal{S}, i}} \oplus \mathbb{Z}^{r_{\Gamma}-r_{\mathcal{S}}}$ where $n_{\mathcal{S}} \geq 0$ and $1<d_{\mathcal{S}, i} \mid d_{\mathcal{S}, i+1}$. The LCM-period $\rho_{\mathcal{A}}$ of $\mathcal{A}$ is defined by

$$
\rho_{\mathcal{A}}:=\operatorname{lcm}\left(d_{\mathcal{S}, n_{\mathcal{S}}} \mid \mathcal{S} \subseteq \mathcal{A}\right) .
$$

It is proved in [BM14] that $\# \mathcal{M}\left(\mathcal{A} ; \Gamma, \mathbb{Z}_{q}\right)$ is a quasi-polynomial in $q \in \mathbb{Z}_{>0}$ for which $\rho_{\mathcal{A}}$ is a period. The quasi-polynomial is called the chromatic quasi-polynomial of $\mathcal{A}$, and denoted by $\chi_{\mathcal{A}}^{\text {quasi }}(q)$. More precisely, there exist polynomials $f_{\mathcal{A}}^{k}(t) \in \mathbb{Z}[t]\left(1 \leq k \leq \rho_{\mathcal{A}}\right)$, called the $k$-constituents, such that

$$
\# \mathcal{M}\left(\mathcal{A} ; \Gamma, \mathbb{Z}_{q}\right)=f_{\mathcal{A}}^{k}(q) \text { if } q \equiv k \bmod \rho_{\mathcal{A}} .
$$

The chromatic quasi-polynomial is precisely the $\mathbb{Z}_{q}$-characteristic polynomial in variable $q$, i.e., $\chi_{\mathcal{A}}^{\text {quasi }}(q)=\chi_{\mathcal{A}}^{\mathbb{Z}_{q}}(q)$ (e.g., [LTY17, Theorem 5.4]), and also the Chen-Wang's quasi-polynomial [Tra18]. In particular, when $\Gamma=\mathbb{Z}^{\ell}$, the $\mathbb{Z}_{q}$-plexification is the $q$-reduced arrangement defined on $\mathcal{A}$ with $\chi_{\mathcal{A}}^{\text {quasi }}(q)$ is the characteristic quasi-polynomial in the sense of [KTT08]. A partial description of the constituents in connection with arrangement theory is known.

Theorem 2.4 ([Tra18]).

$$
f_{\mathcal{A}}^{1}(t)= \begin{cases}0 & \text { if } \mathcal{A}^{\text {tor }} \neq \emptyset, \\ \chi_{\mathcal{A}(\mathbb{R})}(t) & \text { if } \mathcal{A}^{\text {tor }}=\emptyset .\end{cases}
$$

Theorem 2.5 ([LTY17]). Assume that $\Gamma$ is free and $0_{\Gamma} \notin \mathcal{A}$. Then

$$
f_{\mathcal{A}}^{\rho_{\mathcal{A}}}(t)=\chi_{\mathcal{A}}^{\text {toric }}(t) .
$$

\section{THE COMBINATORICS}

Unless otherwise stated, throughout this section, we assume that $G$ is an abelian Lie group with finitely many connected components i.e. $G \simeq$ $\left(\mathbb{S}^{1}\right)^{p} \times \mathbb{R}^{q} \times F$ with $g:=\operatorname{dim}(G)=p+q \geq 0$ and $F$ is a finite abelian group. For each $\mathcal{S} \subseteq \mathcal{A}$, by [LTY17, Proposition 3.6], we have

$$
\begin{aligned}
H_{\mathcal{S}, G} & :=\bigcap_{\alpha \in \mathcal{S}} H_{\alpha, G} \\
& \simeq \operatorname{Hom}\left((\Gamma /\langle\mathcal{S}\rangle)_{\mathrm{tor}}, G\right) \times F^{r_{\Gamma}-r_{\mathcal{S}}} \times\left(\left(\mathbb{S}^{1}\right)^{p} \times \mathbb{R}^{q}\right)^{r_{\Gamma}-r_{\mathcal{S}}} .
\end{aligned}
$$

We agree that $T:=H_{\emptyset, G}$. Each connected component of $H_{\mathcal{S}, G}$ is isomorphic to $\left(\left(\mathbb{S}^{1}\right)^{p} \times \mathbb{R}^{q}\right)^{r_{\Gamma}-r_{\mathcal{S}}}$. If either $r_{\Gamma}=0$ or $g=0$, it can be identified with a point. The set of the connected components of $H_{\mathcal{S}, G}$ is denoted by $\operatorname{cc}\left(H_{\mathcal{S}, G}\right)$. The following lemma is somewhat more general than [Moc12, Lemma 5.4]. 
Lemma 3.1. $\# \operatorname{cc}\left(H_{\mathcal{S}, G}\right)=m(\mathcal{S} ; G) \cdot(\# F)^{r_{\Gamma}-r_{\mathcal{S}}}$.

Most of the main concepts in this section are defined by inspiration of [Moc12, §5] and [LTY17, §7].

\section{Definition 3.2.}

(1) The total intersection poset of $\mathcal{A}(G)$ is defined by

$L=L_{\mathcal{A}(G)}^{\text {tot }}:=\left\{\right.$ connected components of nonempty $\left.H_{\mathcal{S}, G} \mid \mathcal{S} \subseteq \mathcal{A}\right\}$,

whose elements, called layers, are ordered by reverse inclusion $\left(\mathcal{D} \leq_{L}\right.$ $\mathcal{C}$ if $\mathcal{D} \supseteq \mathcal{C}$ ).

(2) The total characteristic polynomial of $\mathcal{A}(G)$ is defined by

$$
\chi_{\mathcal{A}(G)}^{\mathrm{tot}}(t):=\sum_{\mathcal{C} \in L} \mu\left(T^{\mathcal{C}}, \mathcal{C}\right) t^{\operatorname{dim}(\mathcal{C})}
$$

Here $T^{\mathcal{C}}$ is the connected component of $T$ that contains $\mathcal{C}$, and $\mu:=\mu_{L}$.

The set of minimal elements of $L$ is exactly $\operatorname{cc}(T)$. The connected components of $H_{\mathcal{A}, G}$ are maximal elements of $L$ but the converse is not necessarily true. For each $\mathcal{C} \in L$, set

$$
\mathcal{R}(\mathcal{C}):=\left\{\mathcal{S} \subseteq \mathcal{A} \mid \mathcal{C} \in \operatorname{cc}\left(H_{\mathcal{S}, G}\right)\right\}
$$

One observes that $\operatorname{dim}(\mathcal{C})=\operatorname{dim}\left(H_{\mathcal{S}, G}\right)=g\left(r_{\Gamma}-r_{\mathcal{S}}\right)$ for every $\mathcal{S} \in \mathcal{R}(\mathcal{C})$. The localization of $\mathcal{A}$ with respect to $\mathcal{C}$ is defined by

$$
\mathcal{A}_{\mathcal{C}}:=\left\{\alpha \in \mathcal{A} \mid \mathcal{C} \subseteq H_{\alpha, G}\right\}
$$

Stated differently, $\mathcal{A}_{\mathcal{C}}$ is the unique maximal element of $\mathcal{R}(\mathcal{C})$ in the sense that $\mathcal{S} \subseteq \mathcal{A}_{\mathcal{C}}$ for every $\mathcal{S} \in \mathcal{R}(\mathcal{C})$. We also can write

$$
\mathcal{R}(\mathcal{C})=\left\{\mathcal{S} \subseteq \mathcal{A}_{\mathcal{C}} \mid r_{\mathcal{S}}=r_{\mathcal{A}_{\mathcal{C}}}\right\}
$$

Thus $L$ is a ranked poset with a rank function given by $\operatorname{rk}_{L}(\mathcal{C}):=r_{\mathcal{A}_{\mathcal{C}}}=$ $\operatorname{codim}(\mathcal{C}) / g$ for $\mathcal{C} \in L$.

We are interested in a particular subset of $\operatorname{cc}(T)$,

$$
\begin{aligned}
\operatorname{scc}(T) & :=\left\{T_{i} \in \operatorname{cc}(T) \mid\left(\mathcal{A}_{T_{i}}\right)^{\text {tor }}=\emptyset\right\} \\
& =\operatorname{cc}(T) \backslash \bigcup_{\alpha \in \mathcal{A}^{\text {tor }}} \operatorname{cc}\left(H_{\alpha, G}\right) .
\end{aligned}
$$

By using the Inclusion-Exclusion principle,

$$
\# \operatorname{scc}(T)=\# \mathcal{M}\left(\mathcal{A}^{\text {tor }} ; \Gamma_{\text {tor }}, G\right) \cdot(\# F)^{r_{\Gamma}} .
$$

\section{Definition 3.3.}


(1) The partial intersection poset of $\mathcal{A}(G)$ is defined by

$$
L^{\mathrm{par}}:=\left\{\mathcal{C} \in L \mid T^{\mathcal{C}} \in \operatorname{scc}(T)\right\},
$$

with the Möbius function of $L^{\text {par }}$ is the restriction of $\mu$ i.e., $\mu_{L^{\mathrm{par}}}=$ $\left.\mu\right|_{L^{\mathrm{par}} \times L^{\mathrm{par}}}$.

(2) The partial characteristic polynomial of $\mathcal{A}(G)$ is defined by

$$
\chi_{\mathcal{A}(G)}^{\mathrm{par}}(t):=\sum_{\mathcal{C} \in L^{\mathrm{par}}} \mu\left(T^{\mathcal{C}}, \mathcal{C}\right) t^{\operatorname{dim}(\mathcal{C})} .
$$

In other words, $L^{\mathrm{par}}$ is the dual order ideal (e.g., [Sta11, §3.1]) of $L$ generated by $\operatorname{scc}(T)$. It follows from the definition above that $\chi_{\mathcal{A}(G)}^{\mathrm{par}}(t)=0$ if $\operatorname{scc}(T)=\emptyset$.

Remark 3.4. Removing from $\mathcal{A}(G)$ the hyperplanes $H_{\alpha, G}$ with $\alpha \in \mathcal{A}^{\text {tor }}$ does not affect the structure of the poset i.e.,

$$
L_{\mathcal{A}(G)}=L_{\left(\mathcal{A} \backslash \mathcal{A}^{\text {tor }}\right)(G)}=L_{\left(\mathcal{A} \backslash \mathcal{A}^{\text {tor }}\right)(G)}^{\text {par }} .
$$

As a consequence,

$$
\chi_{\mathcal{A}(G)}^{\mathrm{tot}}(t)=\chi_{\left(\mathcal{A} \backslash \mathcal{A}^{\mathrm{tor}}\right)(G)}^{\mathrm{tot}}(t)=\chi_{\left(\mathcal{A} \backslash \mathcal{A}^{\mathrm{tor}}\right)(G)}^{\mathrm{par}}(t) .
$$

In particular, $\chi_{\mathcal{A}(G)}^{\text {tot }}(t)=\chi_{\mathcal{A}(G)}^{\text {par }}(t)$ if $\mathcal{A}^{\text {tor }}=\emptyset$.

In the lemma below, we generalize the result in [Moc12, Lemma 5.5] as we include the possibility $\mathcal{A}^{\text {tor }} \neq \emptyset$.

Lemma 3.5. If $\mathcal{C} \in L$, then

$$
\sum_{\mathcal{S} \in \mathcal{R}(\mathcal{C})}(-1)^{\# \mathcal{S}}=\left\{\begin{array}{l}
\mu\left(T^{\mathcal{C}}, \mathcal{C}\right) \quad \text { if } \mathcal{C} \in L^{\mathrm{par}}, \\
0 \quad \text { if } \mathcal{C} \notin L^{\mathrm{par}}
\end{array}\right.
$$

Proof. The proof of the formula when $\mathcal{C} \in L^{\text {par }}$ is processed by induction on $\mathrm{rk}_{L}(\mathcal{C})$, which runs essentially the same as that of [Moc12, Lemma 5.5]. Note that $\mathcal{A}_{\mathcal{D}} \subseteq \mathcal{A}_{\mathcal{C}}$ whenever $\mathcal{C} \subseteq \mathcal{D} \subseteq T^{\mathcal{C}}$. If $\left(\mathcal{A}_{T^{\mathcal{C}}}\right)^{\text {tor }} \neq \emptyset$ then $\left(\mathcal{A}_{\mathcal{C}}\right)^{\text {tor }} \neq \emptyset$. The remaining part of the formula follows from Theorem 2.4. Indeed, by (3.2)

$$
\sum_{\mathcal{S} \in \mathcal{R}(\mathcal{C})}(-1)^{\# \mathcal{S}}=\sum_{\substack{\mathcal{S} \subseteq \mathcal{A}_{\mathcal{C}} \\ r_{\mathcal{S}}=r_{\mathcal{A}_{\mathcal{C}}}}}(-1)^{\# \mathcal{S}}
$$

equals the coefficient of $t^{r_{\Gamma}-r_{\mathcal{A}_{\mathcal{C}}}}$ in $f_{\mathcal{A}_{\mathcal{C}}}^{1}(t)$, which is 0 .

Corollary 3.6. The Möbius function of L strictly alternates in sign. That is, for all $\mathcal{C} \in L$,

$$
(-1)^{\mathrm{rk}_{L}(\mathcal{C})} \mu\left(T^{\mathcal{C}}, \mathcal{C}\right)>0
$$


Proof. Consider $\mathcal{C} \in L^{\text {par }}$. Note that $f_{\mathcal{A}_{\mathcal{C}}}^{1}(t)=\chi_{\left(\mathcal{A}_{\mathcal{C}}\right)(\mathbb{R})}(t)=\sum_{j=r_{\Gamma}-r_{\mathcal{A}_{\mathcal{C}}}}^{r_{\Gamma}} b_{j} t^{j}$ with $(-1)^{r_{\Gamma}-j} b_{j}>0$ for all $j$ (e.g., [Sta07, Corollary 3.5]). By Proof of Lemma $3.5, \mu\left(T^{\mathcal{C}}, \mathcal{C}\right)$ is equal to the coefficient of $t^{r_{\Gamma}-r_{\mathcal{A}_{\mathcal{C}}}}$ in $f_{\mathcal{A}_{\mathcal{C}}}^{1}(t)$, which strictly alternates in sign i.e., $(-1)^{r} \mathcal{A}_{\mathcal{C}} \mu\left(T^{\mathcal{C}}, \mathcal{C}\right)>0$. If $\mathcal{C} \notin L^{\text {par }}$, we consider $\mathcal{A} \backslash \mathcal{A}^{\text {tor }}$ instead of $\mathcal{A}$ as argued in Remark 3.4.

The main idea of the proof below is very similar to the one used in [Moc12, Theorem 5.6]. We include it with a detailed proof for the sake of completeness.

Theorem 3.7. Let $G \simeq\left(\mathbb{S}^{1}\right)^{p} \times \mathbb{R}^{q} \times F$ with $g=p+q \in \mathbb{Z}_{>0}$. Then

$$
\chi_{\mathcal{A}(G)}^{\mathrm{par}}(t)=\chi_{\mathcal{A}}^{G}\left(\# F \cdot t^{g}\right) .
$$

Proof. We must prove that

$$
\sum_{\mathcal{C} \in L^{\text {par }}} \mu\left(T^{\mathcal{C}}, \mathcal{C}\right) t^{\operatorname{dim}(\mathcal{C})}=\sum_{\mathcal{S} \subseteq \mathcal{A}}(-1)^{\# \mathcal{S}} m(\mathcal{S} ; G) \cdot(\# F)^{r_{\Gamma}-r_{\mathcal{S}}} \cdot t^{g\left(r_{\Gamma}-r_{\mathcal{S}}\right)}
$$

It is equivalent to proving that for all $k=r_{\Gamma}-r_{\mathcal{A}}, \ldots, r_{\Gamma}$,

$$
\sum_{\substack{\mathcal{C} \in L^{\mathrm{par}} \\ g k=\operatorname{dim}(\mathcal{C})}} \mu\left(T^{\mathcal{C}}, \mathcal{C}\right)=\sum_{\substack{\mathcal{S} \subseteq \mathcal{A} \\ k=r_{\Gamma}-r_{\mathcal{S}}}}(-1)^{\# \mathcal{S}} m(\mathcal{S} ; G) \cdot(\# F)^{r_{\Gamma}-r_{\mathcal{S}}}
$$

We have

$$
\begin{aligned}
\sum_{\substack{\mathcal{C} \in L^{\mathrm{par}} \\
g k=\operatorname{dim}(\mathcal{C})}} \mu\left(T^{\mathcal{C}}, \mathcal{C}\right) & =\sum_{\substack{\mathcal{C} \in L \\
g k=\operatorname{dim}(\mathcal{C})}} \sum_{\mathcal{S} \in \mathcal{R}(\mathcal{C})}(-1)^{\# \mathcal{S}} \\
& =\sum_{\substack{\mathcal{S} \subseteq \mathcal{A} \\
r_{\mathcal{S}}=r_{\Gamma}-k}}\left(\sum_{\mathcal{C} \in \operatorname{cc}\left(H_{\mathcal{S}, G}\right)} 1\right)(-1)^{\# \mathcal{S}} \\
& =\sum_{\substack{\mathcal{S} \subseteq \mathcal{A} \\
k=r_{\Gamma}-r_{\mathcal{S}}}}(-1)^{\# \mathcal{S}} m(\mathcal{S} ; G) \cdot(\# F)^{r_{\Gamma}-r_{\mathcal{S}}}
\end{aligned}
$$

We have applied Lemma 3.5 in the first equality, switched roles of sums in the second equality, and used Lemma 3.1 in the last equality.

Corollary 3.8. Let $G \simeq\left(\mathbb{S}^{1}\right)^{p} \times \mathbb{R}^{q} \times F$ with $g=p+q \in \mathbb{Z}_{>0}$. Then

$$
\chi_{\mathcal{A}(G)}^{\text {tot }}(t)=\chi_{\mathcal{A} \backslash \mathcal{A}^{\text {tor }}}^{G}\left(\# F \cdot t^{g}\right) .
$$

Proof. It follows from Theorem 3.7 and Remark 3.4.

Remark 3.9. Although either Theorem 3.7 or Corollary 3.8 may not be valid when $g=0$, there is no loss of information in these formulations. Namely, $\chi_{\mathcal{A}(G)}^{\mathrm{par}}(t)=\# \operatorname{scc}(T)$, and by equality (3.3) and Proposition 2.3, this equals 
the "leading part" of $\chi_{\mathcal{A}}^{G}(\# F)$ (the value of the leading term of $\chi_{\mathcal{A}}^{G}(t)$ evaluated at $\# F)$. Similarly, $\chi_{\mathcal{A}(G)}^{\text {tot }}(t)=\# \mathrm{cc}(T)$, which is equal to the leading part of $\chi_{\mathcal{A} \backslash \mathcal{A}^{\text {tor }}}^{G}(\# F)$.

Remark 3.10. Note that when $G=\mathbb{S}^{1}$ (or $G=\mathbb{C}^{\times}$if the dimension is defined over $\mathbb{C}$ ) and $\mathcal{A}^{\text {tor }}=\emptyset, \chi_{\mathcal{A}(G)}^{\text {par }}(t)=\chi_{\mathcal{A}(G)}^{\text {tot }}(t)=\chi_{\mathcal{A}}^{\text {toric }}(t)$. The result of Moci (Theorem 2.1) is a special case of Corollary 3.8.

\section{THE CONSTITUENTS}

4.1. Via subspace viewpoint. Our first result in this section is the interpretation for chromatic polynomials and their constituents through the real subspace arrangement viewpoint. Combining Theorem 3.7 with the property of the chromatic polynomials (e.g., [Tra18, Proposition 3.6]), we obtain

Corollary 4.1. Let $G=\mathbb{R}^{g} \times \mathbb{Z}_{k}$ with $g>0$ and $1 \leq k \leq \rho_{\mathcal{A}}$. Then

$$
\chi_{\mathcal{A}(G)}^{\mathrm{par}}(t)=f_{\mathcal{A}}^{k}\left(k \cdot t^{g}\right) .
$$

Let us explain Corollary 4.1 in more details. For nontriviality, we assume that $\operatorname{scc}(T) \neq \emptyset$ (e.g., when $\mathcal{A}^{\text {tor }}=\emptyset$ ), and $r_{\Gamma}>0$. Each connected component of $T=\operatorname{Hom}\left(\Gamma, \mathbb{R}^{g} \times \mathbb{Z}_{k}\right)$ is isomorphic to $\mathbb{R}^{g r_{\Gamma}}$. For each $T_{i} \in \operatorname{scc}(T)$, the poset $L_{i}=\left\{\mathcal{C} \in L \mid \mathcal{C} \subseteq T_{i}\right\}$ is isomorphic to the total (or equivalently, partial) intersection poset of a $\mathbb{R}^{g}$-plexification $\mathcal{G}_{i}$ in $\mathbb{R}^{g r_{\Gamma}}$ (or g-plexification in the sense of [Bjö94, §5.2])), with each $\mathcal{G}_{i}$ is possibly empty and defined over the integers. Thus after a rescaling of variable, each constituent records the summation of the total characteristic polynomials of the $\mathcal{G}_{i}$ 's i.e.,

$$
f_{\mathcal{A}}^{k}\left(k t^{q}\right)=\sum_{T_{i} \in \operatorname{scc}(T)} \chi_{\mathcal{G}_{i}}^{\text {tot }}(t) .
$$

Remark 4.2. In particular, when $g=1$, each $\mathcal{G}_{i}$ becomes an integral hyperplane arrangement $\mathcal{H}_{i}$ [Tra18, Proposition 4.5]. The conclusion related to the first constituent $(k=1)$ in Corollary 4.1 is the same as that stated in Theorem 2.4. In particular, if $\Gamma=\mathbb{Z}^{\ell}$, each hyperplane $H_{\alpha, \mathbb{R} \times \mathbb{Z}_{k}}$ in $T$ can be identified with $H_{\alpha, \mathbb{R}} \times H_{\alpha, \mathbb{Z}_{k}}$ in $\mathbb{R}^{\ell} \times \mathbb{Z}_{k}^{\ell}$. Each arrangement $\mathcal{H}_{i}$ turns out to be a subarrangement of $\mathcal{A}(\mathbb{R})$, and in which components of $T$ that the components of $H_{\alpha, \mathbb{R} \times \mathbb{Z}_{k}}$ locate depends on the arithmetics of the list $\mathcal{A}$.

Example 4.3. Let $\Gamma=\mathbb{Z}^{2}, \mathcal{A}=\{\alpha, \beta, \gamma\} \subsetneq \mathbb{Z}^{2}$ with $\alpha=(-1,1), \beta=$ $(0,2)$, and $\gamma=(0,4)$. Then

$$
\chi_{\mathcal{A}}^{\text {quasi }}(q)= \begin{cases}q^{2}-2 q+1 & \text { if } \operatorname{gcd}(q, 4)=1 \\ q^{2}-3 q+2 & \text { if } \operatorname{gcd}(q, 4)=2 \\ q^{2}-5 q+4 & \text { if } \operatorname{gcd}(q, 4)=4\end{cases}
$$


Set $G_{k}:=\mathbb{R} \times \mathbb{Z}_{k}$ with $k \in\{1,2,4\}$. The Hasse diagrams of $L_{\mathcal{A}\left(G_{k}\right)}$ are drawn in Figures 1, 2, 3. The total characteristic polynomials $\chi_{\mathcal{A}\left(G_{k}\right)}^{\text {tot }}(t)$ are computed according to the " $\times n$ ", indicator of the number of isomorphic Hasse diagrams of $L_{i}$ 's.

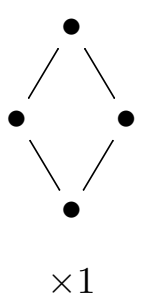

FIGURE 1. $\chi_{\mathcal{A}\left(G_{1}\right)}^{\text {tot }}(t)=t^{2}-2 t+1=f_{\mathcal{A}}^{1}(t)$.
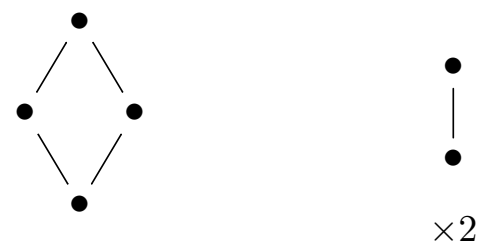

$\times 2$

FIGURE 2. $\chi_{\mathcal{A}\left(G_{2}\right)}^{\mathrm{tot}}(t)=4 t^{2}-6 t+2=f_{\mathcal{A}}^{2}(2 t)$
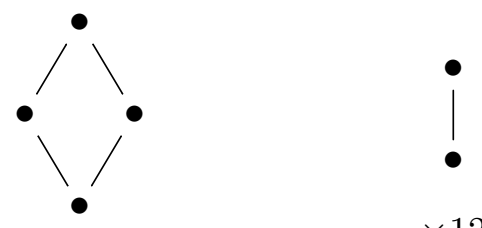

$\times 4$

FIGURE 3. $\chi_{\mathcal{A}\left(G_{4}\right)}^{\text {tot }}(t)=16 t^{2}-20 t+4=f_{\mathcal{A}}^{4}(4 t)$.

Now we give a discussion on reciprocity laws for every constituent $f_{\mathcal{A}}^{k}(t)$. After the works of [CW12] and [Tra18], we know that $(-1)^{r_{\Gamma}} f_{\mathcal{A}}^{k}(-t) \geq 0$ for all $t \in \mathbb{Z}_{>0}$. Also, this fact can be derived from formula (4.6) in this paper. It is natural to ask whether the evaluations $(-1)^{r_{\Gamma}} f_{\mathcal{A}}^{k}(-t)$ have any combinatorial meaning. A partial answer is probably well-known when $\Gamma=\mathbb{Z}^{\ell}$ that $(-1)^{r_{\Gamma}} f_{\mathcal{A}}^{k}(-t)$ can be expressed in terms of the Ehrhart quasipolynomial of an "inside-out" polytope [BZ06]. The construction of the 
polytope and the hyperplanes cutting through it can be found in [KTT08, $\S 2.2]$ (with some modification).

Owning to equality (4.1) and Remark 4.2, we can give an answer to the aforementioned question. For nontriviallity, we assume that $r_{\Gamma}>0$. The reciprocity laws for the characteristic polynomial of an integral arrangement have been formulated by several methods e.g., [Ath10], [BS18, §7], [Wan 15, §4]. Thus the reciprocity laws for any (nonzero) constituent $f_{\mathcal{A}}^{k}(t)$ can be obtained from the reciprocity laws of the polynomials $\chi_{\mathcal{H}_{i}}(t)$ (at least the method from [Ath10] is applicable here) as follows:

$$
(-1)^{r_{\Gamma}} f_{\mathcal{A}}^{k}(-t)=\sum_{i}(-1)^{r_{\Gamma}} \chi_{\mathcal{H}_{i}}\left(\frac{-t}{k}\right) .
$$

4.2. Via toric viewpoint. We may expect that if there exists a "nicer" expression to describe every constituent without making any rescaling of variable. It turns out that such expression can be obtained from the toric arrangement by appropriately extracting its poset of layers. Now let us turn to the second interpretation via toric arrangement viewpoint. In the remainder of this section, we assume that $G$ is either $\mathbb{S}^{1}$ or $\mathbb{C}^{\times}$. We retain the notation of the total group $T=\operatorname{Hom}(\Gamma, G)$, with the identity is denoted by $\mathbf{1}$. For each $k \in \mathbb{Z}$, consider the homomorphism

$$
E_{k}: T \longrightarrow T \text { via } \varphi \mapsto \varphi^{k}:=\varphi \cdots \varphi
$$

\section{Definition 4.4.}

(1) For each $k \in \mathbb{Z}$, the $k$-total intersection poset of $\mathcal{A}(G)$ is defined by

$$
L[k]=\left\{\mathcal{C} \in L \mid \mathbf{1} \in E_{k}(\mathcal{C})\right\} .
$$

(2) The $k$-total characteristic polynomial of $\mathcal{A}(G)$ is defined by

$$
\chi_{\mathcal{A}(G)}^{k \text {-tot }}(t):=\sum_{\mathcal{C} \in L[k]} \mu\left(T^{\mathcal{C}}, \mathcal{C}\right) t^{\operatorname{dim}(\mathcal{C})} .
$$

The cover relation in $L$ is preserved in $L[k]$ i.e., if $\mathcal{C}$ covers $\mathcal{D}$ in $L$ and $\mathcal{C} \in L[k]$ then $\mathcal{D} \in L[k]$, which implies that $L[k]$ is an order ideal (e.g., [Sta11, §3.1]). For each $\mathcal{S} \subseteq \mathcal{A}$, note that $H_{\mathcal{S}, G}$ is a subtorus of $T$ whose each connected component is isomorphic to the torus $G^{r_{\Gamma}-r_{\mathcal{S}}}$. Let $\mathcal{C}_{\mathcal{S}}^{\mathbf{1}} \in \operatorname{cc}\left(H_{\mathcal{S}, G}\right)$ be the identity component of $H_{\mathcal{S}, G}$, that is, the connected component that contains 1 . Thus $\operatorname{cc}\left(H_{\mathcal{S}, G}\right)$ can be identified with the quotient group $H_{\mathcal{S}, G} / \mathcal{C}_{\mathcal{S}}^{1}$. In the lemma below, we generalize [Moc12, Lemma $5.4]$ in an arithmetical manner.

Lemma 4.5. Fix $k \in \mathbb{Z}_{>0}$. For each $\mathcal{S} \subseteq \mathcal{A}$, we have

$$
\#\left(\operatorname{cc}\left(H_{\mathcal{S}, G}\right) \cap L[k]\right)=m\left(\mathcal{S} ; \mathbb{Z}_{k}\right) .
$$


Proof. For each $\mathcal{S} \subseteq \mathcal{A}$, the homomorphism $E_{k}$ induces the endomorphism $\overline{E_{k}}$ of $H_{\mathcal{S}, G} / \mathcal{C}_{\mathcal{S}}^{\mathbf{1}}$ with

$$
\operatorname{ker}\left(\overline{E_{k}}\right)=\operatorname{cc}\left(H_{\mathcal{S}, G}\right) \cap L[k] .
$$

Using the identification $H_{\mathcal{S}, G}=\operatorname{Hom}(\Gamma /\langle\mathcal{S}\rangle, G)$ and a decomposition $\Gamma /\langle\mathcal{S}\rangle=$ $(\Gamma /\langle\mathcal{S}\rangle)_{\text {tor }} \oplus(\Gamma /\langle\mathcal{S}\rangle)_{\text {free, }}$, we can write

$$
\mathcal{C}_{\mathcal{S}}^{\mathbf{1}}=\left\{\varphi \in H_{\mathcal{S}, G} \mid \varphi(x)=1, \forall x \in(\Gamma /\langle\mathcal{S}\rangle)_{\text {tor }}\right\}
$$

Applying the left exact functor $\operatorname{Hom}(-, G)$ to the following exact sequence

$$
0 \longrightarrow\left(\frac{\Gamma}{\langle\mathcal{S}\rangle}\right)_{\text {tor }} \longrightarrow \frac{\Gamma}{\langle\mathcal{S}\rangle} \longrightarrow \frac{\Gamma}{\langle\mathcal{S}\rangle} /\left(\frac{\Gamma}{\langle\mathcal{S}\rangle}\right)_{\text {tor }} \longrightarrow 0
$$

we obtain

$$
H_{\mathcal{S}, G} / \mathcal{C}_{\mathcal{S}}^{\mathbf{1}} \simeq \operatorname{Hom}\left((\Gamma /\langle\mathcal{S}\rangle)_{\text {tor }}, G\right) .
$$

Furthermore, $E_{k}$ induces the endomorphism $\widetilde{E_{k}}$ of $\operatorname{Hom}\left((\Gamma /\langle\mathcal{S}\rangle)_{\text {tor }}, G\right)$ with

$$
\operatorname{ker}\left(\widetilde{E_{k}}\right)=\operatorname{Hom}\left((\Gamma /\langle\mathcal{S}\rangle)_{\text {tor }}, G[k]\right)
$$

Here $G[k]=\left\{x \in G \mid x^{k}=1\right\} \simeq \mathbb{Z}_{k}$. Combining (4.3), (4.4) and (4.5) we get \# $\left(\operatorname{cc}\left(H_{\mathcal{S}, G}\right) \cap L[k]\right)=m\left(\mathcal{S} ; \mathbb{Z}_{k}\right)$, as desired.

\section{Definition 4.6.}

(1) For each $k \in \mathbb{Z}$, the $k$-partial intersection poset of $\mathcal{A}(G)$ is defined by

$$
L^{\text {par }}[k]:=\left\{\mathcal{C} \in L^{\text {par }} \mid \mathbf{1} \in E_{k}(\mathcal{C})\right\} .
$$

(2) The k-partial characteristic polynomial of $\mathcal{A}(G)$ is defined by

$$
\chi_{\mathcal{A}(G)}^{k \text {-par }}(t):=\sum_{\mathcal{C} \in L^{\text {par }}[k]} \mu\left(T^{\mathcal{C}}, \mathcal{C}\right) t^{\operatorname{dim}(\mathcal{C})} .
$$

Theorem 4.7. If $q \in \mathbb{Z}_{>0}$, then

$$
\chi_{\mathcal{A}(G)}^{q-\operatorname{par}}(q)=\chi_{\mathcal{A}}^{\text {quasi }}(q) .
$$

Proof. Very similar to Proof of Theorem 3.7 including the use of Lemma 4.5.

Corollary 4.8. If $1 \leq k \leq \rho_{\mathcal{A}}$, then

$$
\chi_{\mathcal{A}(G)}^{k \text { par }}(t)=f_{\mathcal{A}}^{k}(t) .
$$

Corollary 4.9. If $q \in \mathbb{Z}_{>0}$ and $1 \leq k \leq \rho_{\mathcal{A}}$, then

$$
\begin{aligned}
\chi_{\mathcal{A}(G)}^{q \text {-tot }}(q) & =\chi_{\mathcal{A} \backslash \mathcal{A}^{\text {tor }}}^{\text {quasi }}(q), \\
\chi_{\mathcal{A}(G)}^{k \text {-tot }}(t) & =f_{\mathcal{A} \backslash \mathcal{A}^{\text {tor }}}^{k}(t) .
\end{aligned}
$$


By Theorem 4.7, we can write

$$
\chi_{\mathcal{A}}^{\text {quasi }}(q)=\sum_{j=r_{\Gamma}-r_{\mathcal{A}}}^{r_{\Gamma}}(-1)^{r_{\Gamma}-j} \beta_{j}(q) q^{j},
$$

with each coefficient $\beta_{j}(q)$ is a periodic function given by

$$
\beta_{j}(q)=(-1)^{r_{\Gamma}-j} \sum_{\substack{\mathcal{C} \in L^{\operatorname{par}}(q) \\ j=\operatorname{dim}(\mathcal{C})}} \mu\left(T^{\mathcal{C}}, \mathcal{C}\right) \geq 0 .
$$

It is easily seen that if $a, b \in \mathbb{Z}_{>0}$ and $a \mid b$, then $L^{\text {par }}[a] \subseteq L^{\text {par }}[b]$. This obvious inclusion between the subposets implies the result in [CW12, Theorem 1.2] about the inequality of the constituent coefficients.

Corollary 4.10 ([CW12]). If $a, b$ are positive integers and a divides $b$, then for all $j$ with $r_{\Gamma}-r_{\mathcal{A}} \leq j \leq r_{\Gamma}$,

$$
0 \leq \beta_{j}(a) \leq \beta_{j}(b)
$$

Proof. Note that $\mu$ strictly alternates in sign (Corollary 3.6).

Example 4.11. Let $\Gamma=\mathbb{Z}^{2}, \mathcal{A}=\{\alpha, \beta, \gamma\} \subsetneq \mathbb{Z}^{2}$ with $\alpha=(-1,1), \beta=$ $(0,2)$, and $\gamma=(0,4)$ as in Example 4.3. The Hasse diagrams of $L^{\text {par }}[k]$ are drawn in Figures $4,5,6$. The $k$-partial characteristic polynomials $\chi_{\mathcal{A}(G)}^{k \text {-par }}(t)$ are computed according to the subposets extracted from $L$.

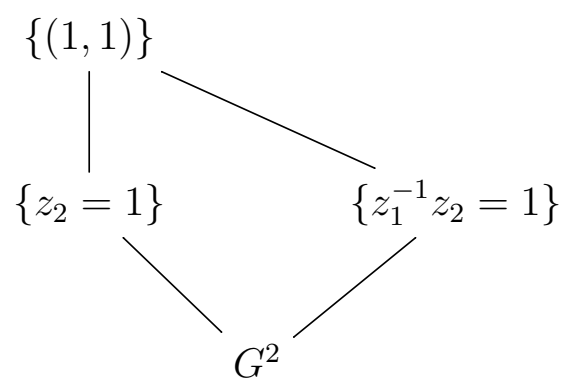

FIGURE 4. $\chi_{\mathcal{A}(G)}^{1-\text { par }}(t)=t^{2}-2 t+1=f_{\mathcal{A}}^{1}(t)$.

Acknowledgements: TNT gratefully acknowledges the support of the scholarship program of the Japanese Ministry of Education, Culture, Sports, Science, and Technology (MEXT) under grant number 142506. MY is partially supported by JSPS KAKENHI Grant Numbers JP16K13741, JP15KK0144, JP18H01115. 


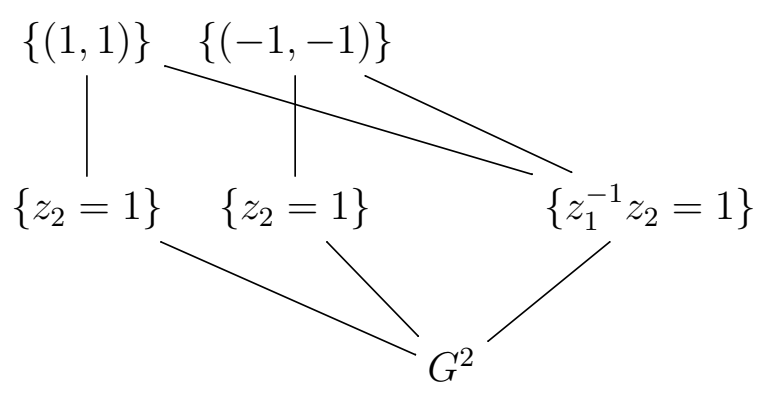

FiguRE 5. $\chi_{\mathcal{A}(G)}^{2-p a r}(t)=t^{2}-3 t+2=f_{\mathcal{A}}^{2}(t)$.

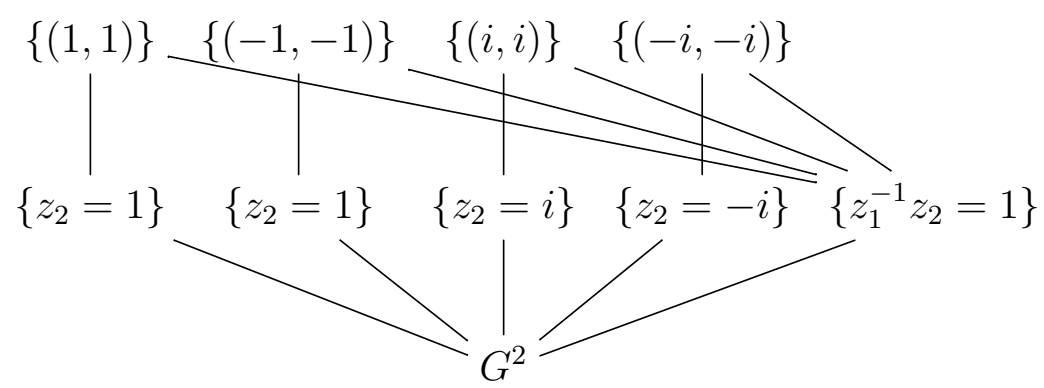

FiguRE 6. $\chi_{\mathcal{A}(G)}^{4-p a r}(t)=t^{2}-5 t+4=f_{\mathcal{A}}^{4}(t)$.

\section{REFERENCES}

[Ath96] C. A. Athanasiadis. Characteristic polynomials of subspace arrangements and finite fields. Adv. Math., 122(2):193-233, 1996.

[Ath10] C. A. Athanasiadis. A combinatorial reciprocity theorem for hyperplane arrangements. Canad. Math. Bull., 53(1):3-10, 2010.

[Bjö94] A. Björner. Subspace arrangements. In: Joseph A., Mignot F., Murat F., Prum B., Rentschler R. (eds) First European Congress of Mathematics. Progress in Mathematics, vol 3. Birkhäuser Basel, 1994.

[BM14] P. Brändén and L. Moci. The multivariate arithmetic Tutte polynomial. Trans. Amer. Math. Soc., 366(10):5523-5540, 2014.

[BS98] A. Blass and B. Sagan. Characteristic and Ehrhart polynomials. J. Algebr. Comb., 7(2):115-126, 1998.

[BS18] M. Beck and R. Sanyal. Combinatorial reciprocity theorems: An invitation to enumerative geometric combinatorics. Graduate Studies in Mathematics, American Mathematical Society, to appear, 2018.

[BZ06] M. Beck and T. Zaslavsky. Inside-out polytopes. Adv. Math., 205(1):134-162, 2006.

[CW12] B. Chen and S. Wang. Comparison on the coefficients of characteristic quasipolynomials of integral arrangements. J. Combin. Theory Ser. A, 119:271-281, 2012. 
[DCP05] C. De Concini and C. Procesi. On the geometry of toric arrangements. Transform. Groups, 10(3-4):387-422, 2005.

[DFM17] C. Dupont, A. Fink, and L. Moci. Universal Tutte characters via combinatorial coalgebras. arXiv preprint, 2017. https://arxiv.org/abs/1711. $09028 \mathrm{v} 1$.

[DM13] M. D'Adderio and L. Moci. Arithmetic matroids, the Tutte polynomial and toric arrangements. Adv. in Math., 232(1):335-367, 2013.

[ERS09] R. Ehrenborg, M. Readdy, and M. Slone. Affine and toric hyperplane arrangements. Discrete Comput. Geom., 41(4):481-512, 2009.

[KTT08] H. Kamiya, A. Takemura, and H. Terao. Periodicity of hyperplane arrangements with integral coefficients modulo positive integers. J. Alg. Combin., 27(3):317330, 2008.

[Law11] J. Lawrence. Enumeration in torus arrangements. European J. Combin., 32(6):870-881, 2011.

[Loo93] E. Looijenga. Cohomology of $\mathcal{M}_{3}$ and $\mathcal{M}_{3}^{1}$. Contemp. Math., 150:205-228, 1993.

[LTY17] Y. Liu, T. N. Tran, and M. Yoshinaga. G-Tutte polynomials and abelian Lie group arrangements. arXiv preprint, 2017. https://arxiv.org/abs/ 1707.04551v2.

[Moc12] L. Moci. A Tutte polynomial for toric arrangements. Trans. Amer. Math. Soc., 364(2):1067-1088, 2012.

[OS80] P. Orlik and L. Solomon. Combinatorics and topology of complements of hyperplane. Invent. Math., 56(1):167-189, 1980.

[OT92] P. Orlik and H. Terao. Arrangements of hyperplanes. Grundlehren der Mathematischen Wissenschaften 300, Springer-Verlag, Berlin, 1992.

[Sta07] R.P. Stanley. An introduction to hyperplane arrangements. in: E. Miller, V. Reiner, B. Sturmfels (Eds.), Geometric Combinatorics, in: IAS/Park City Math. Ser., vol. 13, Amer. Math. Soc., Providence, RI, pp. 389-496, 2007.

[Sta11] R.P. Stanley. Enumerative Combinatorics: Volume 1. Cambridge University Press, New York, NY, USA, 2nd edition, 2011.

[Tra18] T. N. Tran. An equivalent formulation of chromatic quasi-polynomials. arXiv preprint, 2018. https://arxiv.org/abs/1803.08649.

[Tut54] W. T. Tutte. A contribution to the theory of chromatic polynomials. Canad. J. Math., 6:80-91, 1954.

[Wan15] S. Wang. Möbius conjugation and convolution formulae. J. Combin. Theory Ser. $B, 115: 117-131,2015$.

[Yos18a] M. Yoshinaga. Characteristic polynomials of Linial arrangements for exceptional root systems. J. Combin. Theory Ser. A, 157:267-286, 2018.

[Yos18b] M. Yoshinaga. Worpitzky partitions for root systems and characteristic quasipolynomials. Tohoku Math. J., 70(1):39-63, 2018.

Tan Nhat Tran, Department of Mathematics, Hok kaido University, Kita 10, Nishi 8, KiTA-KU, SAPPORO 060-0810, JAPAN.

E-mail address: trannhattan@math.sci.hokudai.ac.jp

Masahiko Yoshinaga, Department of Mathematics, Hokkaido UniverSITY, KITA 10, Nishi 8, KITA-KU, SAPPORO 060-0810, JAPAN.

E-mail address: yoshinaga@math.sci.hokudai.ac.jp 